\title{
Teleportasi Kuantum Terkontrol Keadaan Dua-Qubit Sembarang
}

\author{
Agus Purwanto, ${ }^{*}$ Lila Yuwana, I Nengah Artawan, Bayu Dwi Hatmoko, Rafika Rahmawati, dan Achmad Fatih Alqodri \\ Departemen Fisika-FSains, Institut Teknologi Sepuluh Nopember, Kampus ITS Sukolilo, Surabaya 60111

\section{Intisari}

\begin{abstract}
Kami perlihatkan skema teleportasi terkontrol dua kubit sembarang via kanal lima kubit yang merupakan kombinasi dari keadaan GHZ dan keadaan Bell. 30 dari 32 kombinasi pengukuran oleh Alice dan Charlie memberi transformasi uniter berpasangan, positip-negatip, dan hanya dua yang tidak berpasangan.
\end{abstract}

\begin{abstract}
We have shown in detail a scheme of controlled quantum teleportation of arbitrary two-qubit state via the fivequbit channel which is 30 of 32 measurements of Alice and Charlie give unitary transformations of Bob in positive and negative pairs, the two others are free and different.
\end{abstract}

Keywords: teleportasi kuantum; terkontrol; informasi dua kubit

http://dx.doi.org/10.12962/j24604682.v15i1.4649

2460-4682 (CDepartemen Fisika, Fakultas Sains-ITS

\section{PENDAHULUAN}

Perkembangan teori kuantum saat ini memungkinkan pengembangan teknik informasi berbasis fisika kuantum. Kajian terkait informasi yang mendapat banyak perhatian saat ini adalah teleportasi kuantum yang pertama kali diperkenalkan oleh Bennett dkk [1]. Di dalam skema teoritis teleportasi kuantum, satu keadaan kuantum yang tidak diketahui dikirim melalui pasangan dua partikel berspin $\frac{1}{2}$ dengan bantuan informasi klasik.

Di dalam teleportasi, obyek yang dikirim adalah informasi berupa keadaan kuantum suatu partikel bukan partikelnya itu sendiri. Artinya, dengan teleportasi kita mengirim informasi dari satu partikel ke partikel lain, bukan mengirim materi seperti di dalam film Star Trek.

Sejak diperkenalkan Bennett tahun 1993, kajian aspek teoritis maupun eksperimen teleportasi kuantum terus dilakukan. Eksperimen pertama teleportasi kuantum menggunakan foton terbelit dilakukan oleh Bouwmeester, dkk [2]. Kajian teoritis dapat berupa perluasan kanal tidak lagi pasangan dua partikel tetapi tiga [3] maupun informasi tidak lagi dari partikel tunggal tetapi dua partikel via kanal empat partikel [4]. Teleportasi hanya dapat terjadi via kanal terbelit $[1,5]$ dan mengetahui sifat keterbelitan kubit tinggi menjadi perlu dan telah diakukan [6].

Di artikel ini dikaji teleportasi keadaan dua kubit sembarang terkontrol dengan kanal lima kubit yang merupakan

*Electronic address: purwanto@physics.its.ac.id kajian lebih lanjut dari Shi dkk [7]. Tatanan artikel adalah sebagai berikut. Bagian 1 adalah pendahuluan yakni berupa sejarah singkat teleportasi kuantum. Bagian 2 membahas secara singkat ide awal teleportasi satu kubit dengan kanal dua kubit dan tiga kubit serta perbedaan implikasi dari dua kanal tersebut. Bagian 3 uraian lengkap teleportasi terkontrol keadaan dua kubit via kanal lima kubit dengan semua pengukurannya. Bagian 4 diskusi dan simpulan.

\section{TELEPORTASI QUBIT TUNGGAL}

Di dalam teori kuantum kita mengenal sistem dua keadaan yang di dalam informasi kuantum dirujuk sebagai kuantum bit atau kubit.Contohnya foton terpolarisasi vertikal dan horizontal, keadaan left dan right molekul ammonia $\mathrm{NH}_{3}$, keadaan dasar dan tereksitasi suatu atom atau ion, serta keadaan up dan down partikel dengan spin $\frac{1}{2}$ seperti elektron. Semua ini merealisasi kubit dan secara matematis ekivalen, dan ditulis dalam notasi Dirac standar $|0\rangle$ dan $|1\rangle$.

Konsep awal teleportasi satu kubit dari Benneth dkk adalah sebagai berikut. Alice mempunyai kubit tunggal sembarang yaitu

$$
|\chi\rangle_{a}=x_{\circ}|0\rangle+x_{1}|1\rangle
$$

dengan $\left|x_{\circ}\right|^{2}+\left|x_{1}\right|^{2}=1$. Pada saat yang sama Alice mempunyai kubit kedua yang terbelit dengan kubit ketiga yakni kubit milik Bob dalam bentuk keadaan Bell

$$
|\phi\rangle_{A B}=\frac{1}{\sqrt{2}}(|00\rangle+|11\rangle)
$$


Keadaan yang berperan sebagai kanal ini tidak lain adalah keadaan EPR atau keadaan Bell yang lengkapnya

$$
\begin{aligned}
|b 1\rangle & =\frac{1}{\sqrt{2}}(|00\rangle+|11\rangle) \\
|b 2\rangle & =\frac{1}{\sqrt{2}}(|00\rangle-|11\rangle) \\
|b 3\rangle & =\frac{1}{\sqrt{2}}(|01\rangle+|10\rangle) \\
|b 4\rangle & =\frac{1}{\sqrt{2}}(|01\rangle-|10\rangle)
\end{aligned}
$$

Indeks AB keadaan pada Persamaan (2) menyatakan kubit pertama milik Alice dan kubit kedua milik Bob. Tujuan dari teleportasi adalah mengirim keadaan kubit Alice A pada Persamaan (1) ke kubit Bob B. Keadaan transmisi tidak lain adalah keadaan total dari informasi dan kanal

$$
\begin{aligned}
|\psi\rangle & \equiv|\psi\rangle_{a A B}=|\chi\rangle_{i} \otimes|\phi\rangle \\
& =\frac{x_{\circ}}{\sqrt{2}}(|000\rangle+|011\rangle)+\frac{x_{1}}{\sqrt{2}}(|100\rangle+|111\rangle)
\end{aligned}
$$

Alice kemudian melakukan pengukuran berbasis keadaan Bell pada Persamaan (3) yang secara klasik ditandai sebagai (00, $01,10,11)$ pada dua kubit pertama keadaan pada Persamaan (4), misalkan dengan keadaan

$$
|\Pi\rangle_{a A}=\frac{1}{\sqrt{2}}(|01\rangle+|10\rangle)
$$

Pengukuran tersebut tidak lain adalah proyeksi $\left({ }_{a A}\langle\Pi| \otimes I\right)|\psi\rangle=\left|\psi^{\prime}\right\rangle$.

Selanjutnya Alice melakukan komunikasi klasik dengan Bob menyampaikan pengukurnya (bit 1 dan bit 0) dan Bob melakukan transformasi uniter $\sigma_{B}$ sehingga diperoleh replika dari keadaan Alice (1) pada kubit Bob

$$
|\chi\rangle_{B}=\sigma_{B}\left|\psi^{\prime}\right\rangle=x_{\circ}|0\rangle+x_{1}|1\rangle
$$

dengan $\sigma_{B}=2 \sigma_{x}$. Teleportasi pun selesai.

Pada dasarnya informasi pada Persamaan (1) dapat diteleportasi melalui tiga kanal Bell lainnya. Demikian juga Alice dapat mengukur dengan tiga keadaan Bell lainnya dan mengirimkan informasi pengukurannya ini secara klasik pada Bob sehingga Bob pun melakukan transformasi uniter $\sigma_{B}$ yang sesuai. Kanal dan pengukuran ini masih menggunakan qubit minimal yakni qubit ganda. Informasi pada Persamaan (1) juga dapat diteleportasi melalui kanal tiga kubit, misalkan

$$
|\phi\rangle_{A A B}=\frac{1}{\sqrt{3}}(|001\rangle+|010\rangle+|100\rangle)
$$

Sehingga informasi terbawa dalam kanal $|\psi\rangle_{a A_{1} A_{2} B}=|\chi\rangle_{a} \otimes$

$$
\begin{aligned}
|\chi\rangle_{A_{1} A_{2} B} & \\
|\psi\rangle & \equiv|\psi\rangle_{a A_{1} A_{2} B}=\frac{x_{\circ}}{\sqrt{3}}(|0001\rangle+|0010\rangle+|0100\rangle) \\
& +\frac{x_{1}}{\sqrt{3}}(|1001\rangle+|1010\rangle+|1100\rangle)
\end{aligned}
$$

Selanjutnya, Alice melakukan pengukuran dengan basis

$$
|\Pi\rangle_{a A_{1} A_{2}}=\frac{1}{\sqrt{6}}(2|000\rangle+|010\rangle+|101\rangle)
$$

untuk keadaan pada Persamaan (8) $a A_{1} A_{2}\langle\Pi|\otimes I| \psi\rangle=$ $\left|\psi^{\prime}\right\rangle$. Seperti dalam kasus kanal kubit dua, selanjutnya Alice berkomunikasi secara klasik tentang pengukurnya dan Bob melakukan transformasi uniter $\sigma_{B}$ seperti Persamaan (6) dengan $\left|\psi^{\prime}\right\rangle$ yang sekarang sehingga diperoleh replika pada Persamaan (1) dengan $\sigma_{B}=\frac{3 \sqrt{2}}{2} \sigma_{x}$ dan misi teleportasi selesai.

Tampak bahwa pengukur pada Persamaan (9) lebih rumit dengan tingkat akurasi lebih rendah dibanding tingkat akurasi dari pengukur pada Persamaan (5). Meskipun demikian kanal tiga kubit ini memungkinkan kehadiran seseorang sebagai pengontrol -sebut saja Charlie- dalam teleportasi dan teleportasi dapat terjadi jika ketiganya berinteraksi [3]. Misal, kanal tiga kubit milik Alice sebagai pengirim, Charlie sebagai pengontrol dan Bob sebagai penerima

$$
|\phi\rangle_{A C B}=\frac{1}{\sqrt{2}}(|000\rangle+|111\rangle)
$$

Keadaan total informasi dan kanal $|\psi\rangle_{a A C B}=|\chi\rangle_{a} \otimes$ $|\phi\rangle_{A C B}$

$$
|\psi\rangle=\frac{x_{\circ}}{\sqrt{2}}(|0000\rangle+|0111\rangle)+\frac{x_{1}}{\sqrt{2}}(|1000\rangle+|1111\rangle)(
$$

Alisa mengukur keadaan pada Persamaan (11) ini dengan basis Bell pada Persamaan (5), $\left({ }_{a A}\langle\Pi| \otimes I \otimes I\right)|\psi\rangle$ dan menginformasikan kepada Charlie.

Selanjutnya, Charlie mengukur kubitnya dengan

$$
|\Pi\rangle_{C}=\frac{1}{\sqrt{2}}(|0\rangle+|1\rangle)
$$

dan menginformasikannya kepada Bob. Setelah mendapat informasi dari Charlie, Bob melakukan transformasi uniter pada Persamaan (6) pada kubitnya dengan $\sigma_{B}=2 \sqrt{2} \sigma_{x}$. Replika keadaan pada Persamaan (1) diperoleh dan teleportasi terkontrol selesai.

\section{TELEPORTASI TERKONTROL INFORMASI DUA KUBIT}

Sekarang Alice akan mengirim informasi dua kubit

$$
|\chi\rangle_{a_{1} a_{2}}=x_{\circ}|00\rangle+x_{1}|01\rangle+x_{2}|10\rangle+x_{3}|11\rangle
$$

kepada Bob. Pengiriman ini melibatkan Charlie sebagai pengontrol dan ketiga orang ini mempunyai keadaan terbelit sebagai kanal

$$
\begin{aligned}
|\phi\rangle & \equiv|\phi\rangle_{A_{1} B_{1} C A_{2} B_{2}} \\
& =\frac{1}{2}(|00000\rangle+|00011\rangle+|11100\rangle+|11111\rangle)(
\end{aligned}
$$

Kubit pertama dan keempat dari kanal pada Persamaan (14) adalah milik Alice, kedua dan kelima milik Bob 
dan kubit ketiga milik Charlie. Kanal ini merupakan $\frac{1}{\sqrt{2}}(|00\rangle+|11\rangle)$. kominasi dari dua keadaan terbelit yakni keadaan GHZ $|\phi\rangle_{G H Z}=\frac{1}{\sqrt{2}}(|000\rangle+|111\rangle)$ dan keadaan Bell $|\phi\rangle_{\text {Bell }}=$

Keadaan transmisi yang tidak lain adalah keadaan terlebur total $|\chi\rangle_{a_{1} a_{2}} \otimes|\phi\rangle$

$$
\begin{aligned}
|\psi\rangle & \equiv|\psi\rangle_{a_{1} a_{2} A_{1} B_{1} C A_{2} B_{2}} \\
& =\frac{x_{\circ}}{2}(|0000000\rangle+|0000011\rangle+|0011100\rangle+|0011111\rangle) \\
& +\frac{x_{1}}{2}(|0100000\rangle+|0100011\rangle+|0111100\rangle+|0111111\rangle) \\
& +\frac{x_{2}}{2}(|1000000\rangle+|1000011\rangle+|1011100\rangle+|1011111\rangle) \\
& +\frac{x_{3}}{2}(|1100000\rangle+|1100011\rangle+|1111100\rangle+|1111111\rangle)
\end{aligned}
$$

Mengingat posisi kubit ketiga dan keempat milik Alice serta keenam milik Charlie maka sebelum pengukuran dilakukan operasi perturanan $\mathrm{C}_{\text {int }}$

$$
\begin{aligned}
C_{\text {int }}^{1}= & I \otimes(|00\rangle\langle 00|+| 01\rangle\langle 10|+| 10\rangle\langle 01|+| 11\rangle\langle 11|) \otimes \\
& (|0\rangle I|0\rangle\langle 0|I\langle 0|+| 0\rangle I| 1\rangle\langle 1|I\langle 0|+| 1\rangle I| 0\rangle\langle 0|I\langle 1|+| 1\rangle I| 1\rangle\langle 1| I\langle 1|) \otimes I \\
C_{\text {int }}^{2}= & I \otimes I \otimes I \otimes I \otimes(|00\rangle\langle 00|+| 00\rangle\langle 00|+| 00\rangle\langle 00|+| 00\rangle\langle 00|) \otimes I
\end{aligned}
$$

Sehingga kubit Alice dan Charlie berurutan $|\psi\rangle_{a_{1} A_{1} a_{2} A_{2} C B_{1} B_{2}}$

$$
\begin{aligned}
\left|\psi^{\prime}\right\rangle & =|\psi\rangle_{a_{1} A_{1} a_{2} A_{2} C B_{1} B_{2}} \\
& =C_{\text {int }}^{2} C_{\text {int }}^{1}|\psi\rangle_{a_{1} a_{2} A_{1} A_{2} B_{1} C B_{2}}
\end{aligned}
$$

Pengukuran Alice dengan

$$
\begin{aligned}
\left|\Pi_{a_{1}}\right\rangle & =m_{a}|00\rangle+m_{2}|10\rangle+m_{a}|11\rangle \\
\left|\Pi_{a_{2}}\right\rangle & =n_{a}|00\rangle+n_{2}|10\rangle+n_{a}|11\rangle
\end{aligned}
$$

pada empat keadaan pertama $\left(\left\langle\Pi_{a 1}\left|\otimes\left\langle\Pi_{a 2}\right| \otimes I \otimes I \otimes I\right) \mid \Psi^{\prime}\right\rangle=|\Psi\rangle_{C B_{1} B_{2}}\right.$. Pengukuran ini disampaikan Alice kepada Charlie dan Bob. Selanjutnya Charlie melakukan pengukuran dengan $\left(\left\langle\Pi_{c}\right| \otimes I \otimes I \otimes I\right)|\Psi\rangle_{C B_{1} B_{2}}$

$$
|\Pi\rangle_{C}=a_{\circ}|0\rangle+a_{1}|1\rangle
$$

menghasilkan

$$
\begin{aligned}
|\Pi\rangle_{B_{1} B_{2}} & =a_{\circ}\left(\frac{x_{\circ} m_{\circ} n_{\circ}}{2}+\frac{x_{1} m_{\circ} n_{2}}{2}+\frac{x_{2} m_{2} n_{\circ}}{2}+\frac{x_{3} m_{2} n_{2}}{2}\right)|00\rangle \\
& +a_{\circ}\left(\frac{x_{\circ} m_{\circ} n_{1}}{2}+\frac{x_{1} m_{\circ} n_{3}}{2}+\frac{x_{2} m_{2} n_{1}}{2}+\frac{x_{3} m_{2} n_{3}}{2}\right)|01\rangle \\
& +a_{1}\left(\frac{x_{\circ} m_{1} n_{\circ}}{2}+\frac{x_{1} m_{1} n_{2}}{2}+\frac{x_{2} m_{3} n_{\circ}}{2}+\frac{x_{3} m_{3} n_{2}}{2}\right)|10\rangle \\
& +a_{1}\left(\frac{x_{\circ} m_{1} n_{1}}{2}+\frac{x_{1} m_{1} n_{3}}{2}+\frac{x_{2} m_{3} n_{1}}{2}+\frac{x_{3} m_{3} n_{3}}{2}\right)|11\rangle
\end{aligned}
$$

Selanjutnya Charlie mengirimkan pengukuran ini kepada Bob, dan Bob pun melakukan transformasi uniter pada $|\Psi\rangle_{B_{1} B_{2}}$

$$
|\chi\rangle_{B_{1} B_{2}}=\sigma_{B}|\Psi\rangle_{B_{1} B_{2}}=x_{\circ}|00\rangle+x_{1}|01\rangle+x_{2}|10\rangle+x_{3}|11\rangle
$$

Teleportasi selesai. 
TABEL I: Tabel Pengukuran dan Transformasi Uniter.

\begin{tabular}{|c|c|c|c|}
\hline$\overline{\mid \overline{\left|\Pi_{a_{1}}\right\rangle\left|\Pi_{a_{2}}\right\rangle}}$ & $\left|\Pi_{C}\right\rangle$ & $4 \overline{2} \sqrt{2}|\Psi\rangle_{B_{1} B_{2}}$ & $\sigma_{\beta} / 4 \sqrt{2}$ \\
\hline $\begin{array}{l}0000 \\
0001 \\
0010 \\
0011\end{array}$ & 0 & $\begin{array}{l}x_{\circ}|00\rangle+x_{1}|01\rangle+x_{2}|10\rangle+x_{3}|11\rangle \\
x_{\circ}|00\rangle-x_{1}|01\rangle+x_{2}|10\rangle-x_{3}|11\rangle \\
x_{1}|00\rangle+x_{\circ}|01\rangle+x_{3}|10\rangle+x_{2}|11\rangle \\
-x_{1}|00\rangle+x_{\circ}|01\rangle-x_{3}|10\rangle+x_{2}|11\rangle\end{array}$ & $\begin{array}{l}\mathrm{I} \otimes \mathrm{I} \\
\mathrm{I} \otimes \sigma_{z} \\
\mathrm{I} \otimes \sigma_{x} \\
\mathrm{I} \otimes \mathrm{i} \sigma_{y}\end{array}$ \\
\hline $\begin{array}{l}0000 \\
0001 \\
0010 \\
0011\end{array}$ & 1 & $\begin{array}{l}x_{\circ}|00\rangle+x_{1}|01\rangle-x_{2}|10\rangle-x_{3}|11\rangle \\
x_{\circ}|00\rangle-x_{1}|01\rangle-x_{2}|10\rangle+x_{3}|11\rangle \\
x_{1}|00\rangle+x_{\circ}|01\rangle-x_{3}|10\rangle-x_{2}|11\rangle \\
-x_{1}|00\rangle+x_{\circ}|01\rangle+x_{3}|10\rangle-x_{2}|11\rangle\end{array}$ & $\begin{array}{l}\sigma_{z} \otimes \mathrm{I} \\
\sigma_{z} \otimes \sigma_{z} \\
\sigma_{z} \otimes \sigma_{x} \\
\sigma_{z} \otimes \mathrm{i} \sigma_{y}\end{array}$ \\
\hline $\begin{array}{l}0100 \\
0101 \\
0110 \\
0111 \\
\end{array}$ & 0 & $\begin{array}{l}x_{\circ}|00\rangle+x_{1}|01\rangle-x_{2}|10\rangle-x_{3}|11\rangle \\
x_{\circ}|00\rangle-x_{1}|01\rangle-x_{2}|10\rangle+x_{3}|11\rangle \\
x_{1}|00\rangle+x_{\circ}|01\rangle-x_{3}|10\rangle-x_{2}|11\rangle \\
-x_{1}|00\rangle+x_{\circ}|01\rangle+x_{3}|10\rangle-x_{2}|11\rangle\end{array}$ & $\begin{array}{l}\sigma_{z} \otimes \mathrm{I} \\
\sigma_{z} \otimes \sigma_{z} \\
\sigma_{z} \otimes \sigma_{x} \\
\sigma_{z} \otimes \mathrm{i} \sigma_{y}\end{array}$ \\
\hline $\begin{array}{l}0100 \\
0101 \\
0110 \\
0111\end{array}$ & 1 & $\begin{array}{l}x_{\circ}|00\rangle+x_{1}|01\rangle+x_{2}|10\rangle+x_{3}|11\rangle \\
x_{\circ}|00\rangle-x_{1}|01\rangle+x_{2}|10\rangle-x_{3}|11\rangle \\
x_{1}|00\rangle+x_{\circ}|01\rangle+x_{3}|10\rangle+x_{2}|11\rangle \\
-x_{1}|00\rangle+x_{\circ}|01\rangle-x_{3}|10\rangle+x_{2}|11\rangle\end{array}$ & $\begin{array}{l}\mathrm{I} \otimes \mathrm{I} \\
\mathrm{I} \otimes \sigma_{z} \\
\mathrm{I} \otimes \sigma_{x} \\
\mathrm{I} \otimes \mathrm{i} \sigma_{y} \\
\end{array}$ \\
\hline $\begin{array}{l}1000 \\
1001 \\
1010 \\
1011 \\
\end{array}$ & 0 & $\begin{array}{l}x_{2}|00\rangle+x_{3}|01\rangle+x_{\circ}|10\rangle+x_{1}|11\rangle \\
x_{2}|00\rangle-x_{3}|01\rangle+x_{\circ}|10\rangle-x_{1}|11\rangle \\
x_{3}|00\rangle+x_{2}|01\rangle+x_{1}|10\rangle+x_{\circ}|11\rangle \\
-x_{3}|00\rangle+x_{2}|01\rangle-x_{1}|10\rangle+x_{\circ}|11\rangle\end{array}$ & $\begin{array}{l}\sigma_{x} \otimes \mathrm{I} \\
\sigma_{x} \otimes \sigma_{z} \\
\sigma_{x} \otimes \sigma_{x} \\
\sigma_{x} \otimes \mathrm{i} \sigma_{y}\end{array}$ \\
\hline $\begin{array}{l}1000 \\
1001 \\
1010 \\
1011 \\
\end{array}$ & 1 & $\begin{array}{l}x_{2}|00\rangle+x_{3}|01\rangle-x_{\circ}|10\rangle-x_{1}|11\rangle \\
x_{2}|00\rangle-x_{3}|01\rangle-x_{\circ}|10\rangle+x_{1}|11\rangle \\
x_{3}|00\rangle+x_{2}|01\rangle-x_{1}|10\rangle-x_{\circ}|11\rangle \\
-x_{3}|00\rangle+x_{2}|01\rangle+x_{1}|10\rangle-x_{\circ}|11\rangle\end{array}$ & $\begin{array}{l}\sigma_{z} \otimes \mathrm{I} \\
-\mathrm{i} \sigma_{y} \otimes \sigma_{z} \\
-\mathrm{i} \sigma_{y} \otimes \sigma_{x} \\
\sigma_{y} \otimes \mathrm{i} \sigma_{y} \\
\end{array}$ \\
\hline $\begin{array}{l}1100 \\
1101 \\
1110 \\
1111 \\
\end{array}$ & 0 & $\begin{array}{l}-x_{2}|00\rangle-x_{3}|01\rangle+x_{\circ}|10\rangle+x_{1}|11\rangle \\
-x_{2}|00\rangle+x_{3}|01\rangle+x_{\circ}|10\rangle-x_{1}|11\rangle \\
-x_{3}|00\rangle-x_{2}|01\rangle+x_{1}|10\rangle+x_{\circ}|11\rangle \\
x_{3}|00\rangle-x_{2}|01\rangle-x_{1}|10\rangle+x_{\circ}|11\rangle\end{array}$ & $\begin{array}{l}\mathrm{i} \sigma_{y} \otimes \mathrm{I} \\
\mathrm{i} \sigma_{y} \otimes \sigma_{z} \\
\mathrm{i} \sigma_{y} \otimes \sigma_{x} \\
-\sigma_{y} \otimes \sigma_{y}\end{array}$ \\
\hline $\begin{array}{l}1100 \\
1101 \\
1110 \\
1111\end{array}$ & 1 & $\begin{array}{l}-x_{2}|00\rangle-x_{3}|01\rangle-x_{\circ}|10\rangle-x_{1}|11\rangle \\
-x_{2}|00\rangle+x_{3}|01\rangle-x_{\circ}|10\rangle+x_{1}|11\rangle \\
-x_{3}|00\rangle-x_{2}|01\rangle-x_{1}|10\rangle-x_{\circ}|11\rangle \\
x_{3}|00\rangle-x_{2}|01\rangle+x_{1}|10\rangle-x_{\circ}|11\rangle\end{array}$ & $\begin{array}{l}-\sigma_{x} \otimes \mathrm{I} \\
-\sigma_{x} \otimes \sigma_{z} \\
-\sigma_{x} \otimes \sigma_{x} \\
-\sigma_{y} \otimes \sigma_{y}\end{array}$ \\
\hline
\end{tabular}

Dari bentuk pengukur (18) dan keadaan Bell (3) serta pengukur (19) kita mempunyai 32 kombinasi pengukuran yakni

$$
\begin{aligned}
& m_{\circ}= \pm m_{3}=\frac{1}{\sqrt{2}}, m_{1}=m_{2}=0 \text { dan } m_{\circ}=m_{3}=0, m_{1}= \pm m_{2}=\frac{1}{\sqrt{2}} \\
& n_{\circ}= \pm n_{3}=\frac{1}{\sqrt{2}}, n_{1}=n_{2}=0 \text { dan } n_{\circ}=n_{3}=0, n_{1}= \pm n_{2}=\frac{1}{\sqrt{2}} \\
& a_{\circ}= \pm a_{1}=\frac{1}{\sqrt{2}}
\end{aligned}
$$

Jika bit klasik $a_{\circ}=a_{1}=\frac{1}{\sqrt{2}},\left|\Pi_{c}\right\rangle \rightarrow 0, a_{\circ}=-a_{1}=$ $\frac{1}{\sqrt{2}},\left|\Pi_{c}\right\rangle \rightarrow 1$, dan keadaan Bell seperti diberikan di depan maka semua informasi klasik Alice dan Charlie serta transfor- masi uniter Bob untuk mendapatkan replika informasi Alice diberikan oleh Tabel I. 


\section{SIMPULAN}

Di artikel ini telah diperlihatkan skema teleportasi kuantum terkontrol yakni pengiriman informasi dua kubit sembarang dari Alice kepada Bob di kejauhan dibawah kontrol dari Charlie via kanal lima kubit. Setelah informasi dilebur dalam kanal, keadaan total ditata ulang sedemikian rupa sehingga empat kubit pertama adalah milik Alice, satu kubit berikutnya milik Charlie dan dua kubit terakhir milik Bob. Pengukuran berbasis keadaan Bell oleh Alice dan pengkuran Charlie memberi 32 variasi pengukuran. Pengukuran 00xy1dan 01xy0 memberi hasil yang sama, juga pengukuran 00xy0 dan 01xy1 mempunyai hasil sama. Empat belas dari enambelas pengukuran kubit pertama dan kedua Alice dengan keadaan terbelit Bell ketiga dan keempat menghasilkan transformasi uniter pasangan positip dan negatip, dua lainnya tidak berhubungan.
[1] C.H. Benneth, G. Brassard, C. Crepeau, R. Jozsa, A. Peres, W.K. Wootters, "Teleporting an unknown quantum state via dual classical and Einstein-Podolsky-Rosen channels", Phys.Rev.Lett., vol. 70, pp. 1895-1899, 1993.

[2] D. Bouwmeester, J.W. Pan, K. Mattle, M. Eibl, H. Weinfurter, and A. Zeilinger, "Experimental quantum teleportation", Nature, vol. 390, pp. 575-579, 1997.

[3] A. Karlson, and M. Bourennane, "Quantum teleportation using three-particle entanglement", Phys.Lett. A, vol. 58, no. 6, pp. 4394, 1998.

[4] G. Rigolin, "Quantum teleportation of an arbitrary two-qubit state and its relation to multipartite entanglement", Phys.Rev. A, vol. 71, no. 3, pp. 032303, 2005.
[5] A. Purwanto, H. Sukamto, and L. Yuwana, "Formal Conditions on Quantum Teleportation", Indian J. of Sci. and Tech., vol. 11, no. 18 , pp. 1-6, 2018.

[6] A. Purwanto, H. Sukamto, and L. Yuwana, "Quantum Entanglement and Reduced Density Matrices", Int. J. of Theo. Phys., vol. 57, no. 8, pp. 1-11, 2018.

[7] L. Shi, K. Zhou, J. Wei, Y. Zhu, and Q. Zhu, "Quantum Controlled Teleportation of Arbitrary Two-Qubit State via Entangled States", Advances in Mathematical Physics (Hindawi), volume 2018, Article ID 4575438, 4 pages. [ https://doi.org/10.1155/2018/4575438]. 